UR-1225

ER-13065-678

\title{
Effective Superstrings
}

\author{
Zhu Yang \\ Department of Physics and Astronomy \\ University of Rochester \\ Rochester, NY 14627
}

\begin{abstract}
We generalize the method of quantizing effective strings proposed by Polchinski and Strominger to superstrings. The Ramond-Neveu-Schwarz string is different from the GreenSchwarz string in non-critical dimensions. Both are anomaly-free and Poincare invariant. Some implications of the results are discussed. The formal analogy with 4D (super)gravity is pointed out.
\end{abstract}




\section{Introduction}

Recently Polchinski and Strominger have made substantial progress in quantizing long effective strings such as Nielsen-Olesen vortex and long QCD flux tubes. The essential point is that the measure in path integral approach should be built out of the induced metric rather than the intrinsic metric as used in Polyakov quantization. Such an assumption is physically appropriate for string solitons that arise from underlying well-defined dynamics like the Abelian Higgs model. The results of [1] are likely to have impact on quantization of other effective extended objects. In this note we consider effective string theories that involve fermionic structure on the world sheet. For example, a supersymmetric version of Nielsen-Olesen vortex exists[2]. If we take the case where there is partially broken global supersymmetry[3] with the string soliton, there will be one world-sheet massless fermionic degree of freedom accompanying each transverse oscillation of the string. Such a string is described by a Green-Schwarz (GS) string [4] whose quantization in sub-critical dimension is unexplored. Another case of interest is 3-dimensional Ising model, which has been shown to be a fermionic string. In non-critical region, we expect to consider quantization of an effective fermionic string, possibly Ramond-Neveu-Schwarz (RNS) string [5]. Our approach is incapable of describing the critical behavior, or what is the same, continuum limit, due

to the lack of renormalizability. Finally, we might cite supersymmetric large $N_{c}$ QCD as an example of effective superstring. We will keep in mind these examples when we quantize the effective superstrings.

As pointed out in [1], for these strings none of the standard quantization methods is correct. We will follow closely lines of thought and notation in [1]. The idea is quite simple: whenever there appears the intrinsic Liouville field, we replace it by the induced metric and then check the anomaly cancellation. Field equations are changed and spectrum modified. We first work out the improved superconformal algebra and the spectrum of the 
RNS string, since it is more familiar. Next we quantize GS strings in $D=3,4,6$ and 10 , where they exist. We develop an operator formulation that incorporates the non-trivial feature of conformal anomaly of $\theta$ fields. It turns out that even after GSO projection of the RNS string, the two are not the same, except when $D=10$. Finally we point out some formal analogies of the approach initiated in [1] with other problems in field theory and quantum gravity.

\section{Spinning String}

Locally gauge invariant formulation of a spinning string to the lowest order in derivatives is a $2 \mathrm{D}$ conformal supergravity. It is not known whether it can arise as an effective string from some underlying microscopic model, although there is a conjecture that identifies $\mathrm{D}=3$ spinning string with 3D Ising model. We quantize this string theory because of its elegant structure and for the sake of comparison with the Green-Schwarz string. To describe a long spinning string, if it can exist at all, one should use the induced metric and gravitino. They can be found from the classical equations of motion.

Consider now the RNS string action [6],

$S=-\frac{1}{2 \pi a^{2}} \int d^{2} \sigma e\left\{h^{\alpha \beta} \partial_{\alpha} X^{\mu} \partial_{\beta} X_{\mu}-i \bar{\psi}^{\mu} \rho^{\alpha} \nabla_{\alpha} \psi_{\mu}-2 \bar{\chi}_{\alpha} \rho^{\beta} \rho^{\alpha} \psi^{\mu} \partial_{\beta} X_{\mu}-\frac{1}{2} \psi_{\mu} \psi^{\mu} \bar{\chi}_{\alpha} \rho^{\beta} \rho^{\alpha} \chi_{\beta}\right\}$

which is locally supersymmetric with minimal number of derivatives, thus is the most relevant term of an effective spinning string. Besides general covariance and local supersymmetry, (1) is also invariant under conformal transformation

$$
\delta X^{\mu}=0, \delta \psi^{\mu}=-\frac{1}{2} \Lambda \psi^{\mu}, \delta e_{\alpha}^{a}=\Lambda e_{\alpha}^{a}, \delta \chi_{\alpha}=\frac{1}{2} \chi_{\alpha}
$$

and its super partner

$$
\delta \chi_{\alpha}=i \rho_{\alpha} \eta, \delta e_{\alpha}^{a}=\delta \psi^{\mu}=\delta X^{\mu}=0
$$


In the spirit of [1], the zweibein $e_{\mu}^{a}$ and the gravitino $\chi_{\mu}$ are not independent dynamical variables. Rather they are formed from the matter fields $X_{\mu}$ and $\psi_{\mu}$. As in bosonic string, one should vary $e_{\mu}^{a}$ and $\chi_{\mu}$ and solve them in terms of $X_{\mu}$ and $\psi_{\mu}$. For this purpose we write down the relevant equations of motion,

$$
T_{a}^{\alpha} \equiv \frac{1}{e} \frac{\delta I}{\delta e_{\alpha}^{a}}=0, J_{\alpha}=\frac{1}{2} \partial_{\beta} X_{\mu} \rho^{\beta} \rho^{\alpha} \psi^{\mu}+\frac{1}{4} \bar{\psi}^{\mu} \psi_{\mu} \rho^{\beta} \rho^{\alpha} \chi_{\beta}=0
$$

It is evident from (1) and (4) that we can read off the metric $g_{\mu \nu}$,

$$
g_{\alpha \beta} \sim \partial_{\alpha} X^{\mu} \partial_{\beta} X_{\mu}+\frac{1}{2 i} \bar{\psi}^{\mu} \rho_{(\alpha} \nabla_{\beta)} \psi_{\mu}+\chi-\text { dependence. }
$$

It is however non-trivial to find $\chi_{\mu}$ in general case. As usual things simplify in superconformal gauge, which means in our case

$$
T_{++}=T_{--}=0, J_{\frac{1}{2}+}=J_{-\frac{1}{2}-}=0
$$

where we have switched to light-cone coordinates and $\frac{1}{2}\left(-\frac{1}{2}\right)$ denotes upper (lower) component of a spinor. It is easy to see that in this gauge $g_{++}=g_{--}=0$ and the induced "Liouville" field is

$$
\phi=\ln g_{+-}=\ln \left[\partial_{+} X^{\mu} \partial_{-} X_{\mu}+\psi_{\frac{1}{2}} \partial_{-} \psi_{\frac{1}{2}}+\psi_{-\frac{1}{2}} \partial_{+} \psi_{-\frac{1}{2}}+\chi-\text { dependence }\right]
$$

In order to find its superpartner $\chi$ we use the following trick. A local supersymmetry transformation turns $\phi$ into $\chi$ :

$$
\delta X^{\mu}=\bar{\epsilon} \psi^{\mu}, \delta \psi^{\mu}=-i \rho^{\alpha} \epsilon\left(\partial_{\alpha} X^{\mu}-\bar{\psi}^{\mu} \chi_{\alpha}\right), \delta e_{\alpha}^{a}=-2 i \bar{\epsilon} \rho^{\alpha} \chi_{\alpha}, \delta \chi_{\alpha}=\nabla_{\alpha} \epsilon
$$

Since $\phi$ is determined from (7), we can vary (7) to get $\chi$ in terms of $X_{\mu}$ and $\psi_{\mu}$. They are

$$
\begin{gathered}
\chi_{-\frac{1}{2}} \equiv \chi_{\frac{1}{2}-}=\frac{\partial_{-} \psi_{-\frac{1}{2}}^{\mu} \partial_{+} X_{\mu}}{\partial_{+} X^{\mu} \partial_{-} X_{\mu}}+\cdots \\
\chi_{\frac{1}{2}} \equiv \chi_{-\frac{1}{2}+}=\frac{\partial_{+} \psi_{+\frac{1}{2}}^{\mu} \partial_{-} X_{\mu}}{\partial_{+} X^{\mu} \partial_{-} X_{\mu}}+\cdots
\end{gathered}
$$


In deriving (9) we have omitted higher orders in fermion fields and used the $\chi$-dependent terms in (7) to cancel some unwanted terms linear in $\psi^{\mu}$. By the way, a superspace formulation may facilitate our derivations. We will stick to component form in this paper.

With these preliminary findings we are in a position to compute anomalies. It is wellknown that the Polyakov determinant [0, 8] in terms of intrinsic $\phi$ and $\chi$ induces the following action

$$
S_{L}=\frac{D-10}{8 \pi} \int d^{2} \sigma\left[\partial_{+} \phi \partial_{-} \phi+\chi_{\frac{1}{2}} \partial_{-} \chi_{\frac{1}{2}}+\chi_{-\frac{1}{2}} \partial_{+} \chi_{-\frac{1}{2}}\right]
$$

Now following Polchinski and Strominger, who in turn used an idea of DDK 9], we simply substitute (7) and (9) into (10) and get

$$
\begin{aligned}
S_{L} & =\frac{\beta}{\pi} \int d^{2} \sigma\left[\frac{\partial_{+}^{2} X \cdot \partial_{-} X \partial_{-}^{2} X \cdot \partial_{+} X}{\left(\partial_{+} X \cdot \partial_{-} X\right)^{2}}+\frac{\partial_{+} \psi_{\frac{1}{2}} \cdot \partial_{-}^{2} X}{\left(\partial_{+} X \cdot \partial_{-} X\right)^{2}} \partial_{+} \psi_{\frac{1}{2}} \cdot \partial_{-} X\right. \\
& \left.+\frac{\partial_{-} \psi_{-\frac{1}{2}} \cdot \partial_{+}^{2} X}{\left(\partial_{+} X \cdot \partial_{-} X\right)^{2}} \partial_{-} \psi_{-\frac{1}{2}} \cdot \partial_{+} X\right] .
\end{aligned}
$$

As is the case in [1], the expectation value of $\partial_{+} X \cdot \partial_{-} X$ must be non-zero and large compared to $a^{2}$, i.e., we are dealing with a long string. Note in (11) we have introduced a new coupling constant $\beta$ which will be determined by the anomaly-free condition. Now our problem is transformed into investigating the dynamics generated by (1) plus (11).

In order to stabilize our long string, we follow [1] to periodically identify space in the $X^{1}$ direction with a large radius $R$ and consider strings with winding number 1 ,

$$
X^{\mu}(\tau, \sigma+2 \pi)=X^{\mu}(\tau, \sigma)+2 \pi R \delta_{1}^{\mu}
$$

The classical background is

$$
X_{c l}=e_{+}^{\mu} R \sigma^{+}+e_{-}^{\mu} R \sigma^{-}, \psi_{c l}=0,
$$

where $e_{+} \cdot e_{+}=e_{-} \cdot e_{-}=0$ and $e_{+} \cdot e_{-}=-1 / 2$. We then make an expansion in powers of 
$R^{-1}$. Write $Y^{\mu}=X^{\mu}-X_{c l}^{\mu}$, we find to the next to leading order on $R^{-1}$

$L=-\frac{R^{2}}{8 \pi a^{2}}+\frac{1}{4 \pi a^{2}} \partial_{+} Y \cdot \partial_{-} Y+\frac{1}{2 \pi} \psi_{\frac{1}{2}} \partial_{-} \psi_{\frac{1}{2}}+\frac{1}{2 \pi} \psi_{-\frac{1}{2}} \partial_{+} \psi_{-\frac{1}{2}}+\frac{\beta}{\pi R^{2}} \partial_{+}^{2} Y \cdot e_{-} e_{+} \cdot \partial_{-}^{2} Y+O\left(R^{-3}\right)$.

Note that the fermionic terms in (11) do not contribute in this order. Although not obvious, (14) still possesses superconformal invariance order to order in $R^{-1}$. For convenience we will first compute the modified (super) stress tensor, then use operator product expansion (OPE) to obtain new transformation laws for the matter fields. The superstress tensor can be simply read off from the expression of the intrinsic Liouville contribution. According to [10], it is

$$
\begin{aligned}
T_{++} & =\frac{1}{2} \partial_{+} X \cdot \partial_{+} X+\frac{1}{2} \psi_{\frac{1}{2}} \partial_{+} \psi_{\frac{1}{2}}+\frac{\beta}{2}\left(\partial_{+} \phi \partial_{+} \phi+\partial_{+}^{2} \phi+\chi_{\frac{1}{2}} \partial_{+} \chi_{\frac{1}{2}}\right) \\
G_{\frac{3}{2}} & =\frac{1}{2} \partial_{+} X \cdot \psi_{\frac{1}{2}}+\frac{\beta}{2}\left(\partial_{+} \chi+\partial_{+} \phi \chi_{\frac{1}{2}}\right) .
\end{aligned}
$$

Now we substitute (7) and (9) into the above equation and get

$$
\begin{aligned}
T_{++} & =-\frac{R}{a^{2}} e_{+} \cdot \partial_{+} Y-\frac{1}{2} \partial_{+} Y \cdot \partial_{+} Y+\frac{1}{2} \psi_{\frac{1}{2}} \partial_{+} \psi_{\frac{1}{2}}-\frac{2 \beta}{R} e_{-} \cdot \partial_{+}^{3} Y, \\
G_{\frac{3}{2}} & =\frac{1}{2} R e_{+} \cdot \psi_{\frac{1}{2}}+\frac{1}{2} \partial_{+} Y \cdot \psi_{\frac{1}{2}}+\frac{2 \beta}{R} e_{-} \cdot \partial_{+}^{2} \psi_{\frac{1}{2}}+\left(R^{-1}\right) .
\end{aligned}
$$

Note that to order $R^{-1}$ only the improvement term contributes. This procedure of getting $T_{++}$and $G_{\frac{3}{2}}$ resembles the so-called 1.5 order formalism of supergravity [11]. It is then straightforward to evaluate the OPEs of stress tensor and its superpartner. We obtain, to order $R^{0}$,

$$
\begin{aligned}
T_{++}\left(\sigma^{+}\right) T_{++}(0) & =\frac{3}{4} \frac{D-8 \beta}{\left(\sigma^{+}\right)^{4}}+\frac{2}{\left(\sigma^{+}\right)^{2}} T_{++}(0)+\frac{1}{\sigma^{+}} \partial_{+} T_{++}(0)+O\left(R^{-1}\right), \\
T_{++}\left(\sigma^{+}\right) G_{\frac{3}{2}}(0) & =\frac{3}{2} \frac{1}{\left(\sigma^{+}\right)^{2}} G_{\frac{3}{2}}(0)+\frac{1}{\sigma^{+}} \partial_{+} G_{\frac{3}{2}}(0)+O\left(R^{-1}\right) \\
G_{\frac{3}{2}}\left(\sigma^{+}\right) G_{\frac{3}{2}}(0) & =\frac{D-8 \beta}{4\left(\sigma^{+}\right)^{3}}+\frac{1}{2 \sigma^{+}} T_{++}(0)+O\left(R^{-1}\right) .
\end{aligned}
$$


If $\beta=(D-10) / 8$, the anomaly in (17) cancels the ghost contribution. As in bosonic case, $\beta$ is not renormalized. As usual, the superconformal transformation law of $X^{\mu}$ and $\psi^{\mu}$ is found from the OPE between them and T and G. Since

$$
\begin{aligned}
T_{++}\left(\sigma^{+}\right) Y^{\mu}(0) & =\frac{1}{\sigma}\left(R e_{+}^{\mu}+\partial_{+} Y^{\mu}\right)+\frac{1}{\left(\sigma^{+}\right)^{3}} \frac{2 \beta e_{-}^{\mu}}{R}+\cdots, T_{++}\left(\sigma^{+}\right) \psi_{\frac{1}{2}}^{\mu}(0)=\frac{1}{2 \sigma^{+}} \psi_{\frac{1}{2}}^{\mu}+\cdots, \\
G_{\frac{3}{2}}\left(\sigma^{+}\right) Y^{\mu}(0) & =\frac{1}{2 \sigma^{+}} \psi_{\frac{1}{2}}^{\mu}+\cdots, G_{\frac{3}{2}}\left(\sigma^{+}\right) \psi_{\frac{1}{2}}^{\mu}(0)=\frac{1}{2 \sigma^{+}}\left(R e_{+}^{\mu}+\partial_{+} Y^{\mu}\right)+\frac{1}{\left(\sigma^{+}\right)^{3}} \frac{4 \beta e_{-}^{\mu}}{R},(18)
\end{aligned}
$$

we have, under left-moving (super) conformal transformation $\epsilon^{+}\left(\sigma^{+}\right)$, and $\eta_{-\frac{1}{2}}\left(\sigma^{+}\right)$,

$$
\begin{aligned}
\delta Y^{\mu} & =\epsilon^{+}\left(R e_{+}^{\mu}+\partial_{+} Y^{\mu}\right)-\frac{\beta}{R} \partial_{+}^{2} \epsilon^{+} e_{-}^{\mu}+\frac{1}{2} \eta_{-\frac{1}{2}} \psi_{\frac{1}{2}} \\
\delta \psi_{\frac{1}{2}}^{\mu} & =\frac{1}{2} \partial_{+} \epsilon^{+} \psi_{\frac{1}{2}}^{\mu}+\epsilon^{+} \partial_{+} \psi_{\frac{1}{2}}^{\mu}+\eta_{-\frac{1}{2}}\left(R e_{+}^{\mu}+\partial_{+} Y^{\mu}\right)+\frac{2 \beta}{R} \partial_{+}^{2} \eta_{-\frac{1}{2}} e_{-}^{\mu} .
\end{aligned}
$$

We now examine the spectrum. The bosonic oscillations $Y^{\mu}$ have harmonic expansion with periodic boundary conditions,

$$
\partial_{+} Y=a \sum_{n=-\infty}^{+\infty} \alpha_{n}^{\mu} e^{-i n \sigma^{+}} .
$$

For $\psi^{\mu}$, there are two different expansions as usual: one with periodic boundary condition ( $\mathrm{R}$ sector)

$$
\psi_{+}^{\mu}=\frac{a}{\sqrt{2}} \sum_{n \in Z} d_{n}^{\mu} e^{-i n \sigma^{+}},
$$

and one with antiperiodic condition (NS sector)

$$
\psi_{+}^{\mu}=\frac{a}{\sqrt{2}} \sum_{n \in Z+\frac{1}{2}} d_{n}^{\mu} e^{-i n \sigma^{+}},
$$

Insert the above into the mode expansion of $T$ and $G$ we have the superconformal generators

$$
\begin{aligned}
L_{n} & =\frac{R}{a} e_{+} \cdot \alpha_{n}+\frac{1}{2} \sum_{m \in Z}: \alpha_{n-m} \alpha_{m}:-\frac{\beta}{R} a n^{2} e_{-} \cdot \alpha_{n}+L_{m}^{f} \\
G_{r} & =R e_{+} \cdot b_{r}+\sum_{n=-\infty}^{\infty} \alpha_{-n} \cdot b_{r+n}+\frac{8 \beta r^{2}}{R} e_{-} \cdot b_{r} \\
F_{m} & =R e_{+} \cdot d_{m}+\sum_{n=-\infty}^{\infty} \alpha_{-n} \cdot d_{m+n}+\frac{8 \beta m^{2}}{R} e_{-} \cdot d_{m}
\end{aligned}
$$


where $f=b, d$ denotes NS, R sector contribution to $L_{n}$, respectively:

$$
\begin{aligned}
L_{n}^{b} & =\frac{1}{2} \sum_{r=-\infty}^{\infty}\left(r+\frac{1}{2} n\right): b_{-r} \cdot b_{n+r}:-\frac{\beta}{2} \delta_{n, 0}, \\
L_{n}^{d} & =\frac{1}{2} \sum_{m=-\infty}^{\infty}\left(m+\frac{1}{2} n\right): b_{-n} \cdot b_{m+n}:
\end{aligned}
$$

They satisfy the superconformal algebra with central charge 10,

$$
\begin{aligned}
{\left[L_{m}, L_{n}\right] } & =(m-n) L_{m+n}+A(m) \delta_{m, 0} \\
{\left[L_{m}, G_{r}\right] } & =\left(\frac{1}{2} m-r\right) G_{m+r} \\
\left\{G_{r}, G_{s}\right\} & =2 L_{r+s}+B(r) \delta_{r+s, 0}
\end{aligned}
$$

where

$$
A(m)=\frac{5}{4} m\left(m^{2}-1\right), B(r)=5\left(r^{2}-\frac{1}{4}\right)
$$

For $R$ sector, we replace $G_{r}$ by $F_{n}$ and similar structure emerges with

$$
A(m)=\frac{5}{4} m^{3}, B(n)=5 n^{2}
$$

Now we discuss the ground state. It is well-known that the tachyonic ground state of fundamental RNS string in critical dimension $D=10$ and half of the excited states are inconsistent with modular invariance and thus must be projected out by GSO projection[12]. As a bonus space-time supersymmetry is realized. Is it necessary to do GSO projection for effective spinning string? In the case of Nielsen-Olesen string or its supersymmetric generalization, the string is stable. Interaction like breaking and joining are not allowed. In this case, presumably GSO projection is not necessary. If string interaction exists, one must be prepared to do GSO projection to achieve consistency. In any case, before the GSO projection, the on-shell condition for the NS sector is

$$
G_{r}|\phi\rangle=0(r>0), L_{n}|\phi\rangle=0(n>0),\left(L_{0}-\frac{1}{2}\right)|\phi\rangle=0
$$


while for the $\mathrm{R}$ sector it is

$$
F_{n}|\phi\rangle=L_{n}|\phi\rangle=0(n \geq 0) .
$$

They are the same as for critical strings because they are consequences of, say, BRST invariance [13]. For the NS ground state $|k, k\rangle$, the total momentum is

$$
p^{\mu}=\frac{R}{2 a^{2}}\left(e_{+}^{\mu}+e_{-}^{\mu}\right)+\frac{1}{2}\left(\alpha_{0}^{\mu}+\tilde{\alpha}_{0}^{\mu}\right) .
$$

From the on-shell condition (25) we have $k^{1}=0$. So the mass is

$$
m=\frac{R}{2 a^{2}}-\frac{D-2}{8 R} .
$$

It is a scalar. For $\mathrm{R}$ sector, the ground state energy is simply $R$. The reason is that to the leading order, there is no quantum correction to $F_{0}$.

It is instructive to work out the first excited states in NS sector and observe whether they pair with $\mathrm{R}$ ground state to form a supermultiplet. Let's look at the following state

$$
|\phi\rangle=\epsilon \cdot b_{-\frac{1}{2}} \tilde{\epsilon} \cdot \tilde{b}_{-\frac{1}{2}}|k, \tilde{k} ; 0\rangle
$$

where $\epsilon^{\mu}$ and $\tilde{\epsilon}^{\mu}$ are the polarization vectors. The $G_{\frac{1}{2}}|\phi\rangle=0$ implies that $\epsilon \cdot v=0$, where $v^{\mu}=R e_{+}^{\mu}+k^{\mu}+\beta e_{-}^{\mu} / 4 R$. From $\left(L_{0}-1 / 2\right)|\phi\rangle=0$ we have, $v^{\mu} v_{\mu}=0$. The two conditions imply that there are $D-2$ physical components for each $\epsilon$ and $\tilde{\epsilon}$. The rest mass is $\sqrt{-p^{2}}=R / 2 a^{2}-(D-10) / 8 R$, which is different from the $\mathrm{R}$ ground state. This suggests that there is no space-time supersymmetry in transverse directions even if we take the GSO projection, except for $D=10$. In the next section we will study Green-Schwarz string, where transverse space supersymmetry survives quantization. It is somewhat surprising that the two formulations of fermionic string differ in non-critical dimensions. 


\section{Green-Schwarz superstring}

The Green-Schwarz string describes space-time supersymmetric string. In covariant formulation, the action reads [4

$$
\begin{aligned}
S & =-\frac{1}{2} \int d^{2} \sigma\left\{\sqrt{g} g^{\alpha \beta} \Pi_{\alpha} \cdot \Pi_{\beta}++2 i \epsilon^{\alpha \beta} \partial_{\alpha} X^{\mu}\left(\bar{\theta}^{1} \Gamma_{\mu} \theta^{1}-\bar{\theta}^{2} \Gamma_{\mu} \theta^{2}\right)\right. \\
& \left.+\epsilon^{\alpha \beta} \bar{\theta}^{1} \Gamma^{\mu} \partial_{\alpha} \theta^{1} \bar{\theta}^{2} \Gamma_{\mu} \partial_{\beta} \theta^{2}\right\}, \\
\Pi_{\alpha}^{\mu} & =\partial_{\alpha} X^{\mu}-i \bar{\theta}^{A} \Gamma^{\mu} \partial_{\alpha} \theta^{A} .
\end{aligned}
$$

The nonlinearity and peculiar constraints have made an straightforward covariant quantization impossible. In fact GS string is most thoroughly studied only in light-cone gauge operator formulation, where the modification of the theory in non-critical dimension is not known. A world-sheet covariant path integral method has been developed and anomaly cancellation mechanism is understood[14, 15]. Very little is known, however, about the non-critical GS string. In view of the weak coupling supersymmetric Abelian Higgs model, however, a consistent quantization of a long string definitely exists. Moreover, the powerful method of conformal field theory has not been applied to the GS string. Of course the path integral approach and CFT must be equivalent, the latter is however easier to handle. In what follows, we will show how the conformal anomaly arises and differs from familiar

first order systems in the fermion sector in the language of OPE, and how to construct an anomaly-free non-critical theory. As we will see, the interaction between bosons and fermions is essential.

For a long string, a non-covariant gauge fixing in [3, 14, 15] is natural and satisfactory because the string itself breaks Lorentz invariance spontaneously. The gauge fixing is

$$
\gamma^{+} \theta^{A}=0, \gamma_{\alpha \beta}=e^{\phi} \eta_{\alpha \beta}
$$

The first one in (34) fixes the Siegel $\kappa$-symmetry[16]. In this gauge the classical action (33) 
simplifies to

$$
S=\int d^{2} \sigma\left\{\frac{1}{2} \partial_{+} X^{\mu} \partial_{-} X_{\mu}+\bar{\theta}^{1} \gamma^{-} \partial_{+} X^{+} \partial_{-} \theta^{1}+\bar{\theta}^{2} \gamma^{-} \partial_{-} X^{+} \partial_{+} \theta^{2}\right\}
$$

The stress tensor is

$$
T_{++}=\frac{1}{2} \Pi_{+} \cdot \Pi_{+} \cdot
$$

As it stands, $T_{++}$is not well-defined quantum mechanically, due to short distance singularity. Conventionally one normal orders the product of two operators. In case of interacting fields or in the presence of external fields, the normal ordering usually produces finite terms called anomaly. This happens to $T_{++}$too. We normal order one of the terms in (36), $\partial_{+} X^{\mu} \bar{\theta} \gamma^{\mu} \partial_{+} \theta$, since it is the only relevant one contributing to conformal anomaly. The $\theta^{1}$ propagator reads

$$
\bar{\theta}^{1}\left(\sigma^{+}\right) \theta^{1}(0)=\frac{1}{2 \gamma^{-} \sqrt{\partial_{+} X^{+}\left(\sigma^{+}\right)} \sigma^{+} \sqrt{\partial_{+} X^{+}(0)}},
$$

where $\gamma^{-}$is invertible in the subspace of $\gamma^{+} \theta^{1}=0$. The particular form of (37) is chosen to be consistent with general covariance. We must replace $\partial_{+} X^{\mu} \bar{\theta}^{1} \gamma^{\mu} \partial_{+} \theta^{1}$ by

$$
\partial_{+} X^{\mu}: \bar{\theta} \gamma^{\mu} \partial_{+} \theta:-\frac{N_{f}}{8} \frac{\partial_{+} X^{+} \partial_{+}^{3} X^{+}}{\left(\partial_{+} X^{+}\right)^{2}}+O\left(R^{-2}\right)
$$

where where as before $\partial_{+} X^{+} \sim R$ and $N_{f}$ is number of propagating $\theta$ components. Here the normal product simply means that there is no contraction between fields inside it. The significance of the anomalous term in (38) is that it will produce extra conformal anomaly for $\theta$, because we are effectively treating $\theta$ as if it had conformal dimension $1 / 2$, but actually it has dimension 0. Another noteworthy feature of it is its resemblance to the quantum contribution to the stress tensor (16). The normal ordering of other terms in (36) makes no contribution to the leading order. Now we write $T_{++}$we have got so far, again in the background (12) and (13),

$$
T_{++}=-\frac{R}{a^{2}} e_{+} \cdot \partial_{+} Y-\frac{1}{2} \partial_{+} Y \cdot \partial_{+} Y+e_{+}^{\mu}: \bar{\theta}^{1} \gamma^{\mu} \partial_{+} \theta^{1}:-\frac{N_{f}}{8} \frac{1}{R e_{+} \cdot u} \partial_{+}^{3} Y^{+}+O\left(R^{-2}\right),
$$


where $u^{\mu}$ is the unit vector in $X^{+}$direction. If we compute the OPE of $T_{++}$'s we find that the anomaly is

$$
c=D+2 N_{f}
$$

Here, $D$ is of course the $X^{\mu}$ contribution. $N_{f} / 2$ is from direct $\theta$ contraction, and the remaining $3 N_{f} / 2$ is due to the contraction between the first and the last term of (39). For $D=10,6,4,3, N_{f}=8,4,2,1$, respectively. Now follow the previous treatment [1], we add to $T_{++}$a "Liouville" contribution $\beta e_{-} \cdot \partial_{+}^{3} / R$. The complete quantum $T_{++}$then reads, up to $O\left(R^{-2}\right)$,

$T_{++}=-\frac{R}{a^{2}} e_{+} \cdot \partial_{+} Y-\frac{1}{2} \partial_{+} Y \cdot \partial_{+} Y+e_{+}^{\mu}: \theta^{1} \gamma^{\mu} \partial_{+} \theta^{1}:+c \frac{1}{R e_{+} \cdot u} \partial_{+}^{3} Y^{+}+\frac{\beta}{R} e_{-} \cdot \partial_{+}^{3} Y+O\left(R^{-2}\right)$,

where $\beta=(26-c) / 12$. As before, one can check that the anomaly adds up to 26 . Thus we have constructed an anomaly-free effective Green-Schwarz string. In particular, $\beta=0$ for $D=10$. The path integral evaluation of conformal anomaly in critical GS string [14, 15] is recovered.

Let's examine the spectrum. It is straightforward to make mode expansion of $T_{++}$in terms of $L_{n}$ and obtain the Virasoro algebra with central charge 26. As usual, a physical state $|\phi\rangle$ is defined as

$$
L_{n}|\phi\rangle=0(n>0), \quad\left(L_{0}-1\right)|\phi\rangle=0
$$

An important issue is to count the contribution of Casimir effect to $L_{0}$. We have $D / 24$ from $D$ bosons, and -2 from the reparametrization ghosts. For a closed string, the only boundary condition for $\theta$ is periodicity,

$$
\theta^{A}(\sigma, \tau)=\theta^{A}(\sigma+2 \pi, \tau)
$$

So

$$
\theta^{1}=\sum_{n \in Z} \theta_{n}^{1} e^{-2 i n \sigma^{+}} .
$$


Since a periodic fermion has Casimir energy $-1 / 24$, we have $-N_{f} / 24$ from $\theta$. For $D=$ 3, 4, 6, 10, the Casimir energy all cancels. Thus we write

$$
L_{0}=\frac{R}{a} \epsilon_{+} \cdot \alpha_{0}+\frac{1}{2} \sum_{m \in Z}: \alpha_{-m} \cdot \alpha_{m}:+e_{+}^{+} \sum_{m \in Z}: n \bar{\theta}_{-n} \gamma^{-} \theta_{n}:+O\left(R^{-2}\right) .
$$

The ground state thus has mass $R$, as the R sector of RNS string. The ground state forms representations of the $\theta$ zero modes, which is expected to generate supersymmetry in the transverse direction. We leave the details for further study. To conclude this section, we have achieved a world-sheet covariant quantization of non-critical Green-Schwarz string.

The ground state energy is not modified by quantum effects, at least to the leading nontrivial order we are studying. This suggests that the transverse supersymmetry persists after quantization.

\section{Discussion}

A. As we have seen the RNS and GS strings differ from each other when $D \neq 10$. This can be understood in terms of degrees of freedom counting. Th ground state energy (31) of the NS sector is a simple consequence of transversality of physical excitations. Since the Regge slope does not depend on $D$, the quantum correction can not vanish for every $D$. On the other hand, in GS string, the number of bosonic physical degrees of freedom $(D-2)$ equals exactly that of fermions, resulting the cancellation in (45). If we use light-cone gauge quantization, the equivalence proof of the RNS and GS strings in [17] must break down in $D \neq 10$, possibly due to the lack of triality of $\operatorname{Spin}(D-2)$.

B. The continuum limits of the two strings, it they exist, must be different. This raises an interesting question concerning the continuum limit of $3 D$ Ising model. It is known that the $3 D$ Ising model can be represented as a Fermionic string theory. For aesthetic reason and simplicity, one conjectures that the string should have maximal symmetry. This leads to RNS and GS strings. The question is then, which one describes the Ising model? 
C. Kutasov and Seiberg [18] have constructed tachyon-free RNS strings with the intrinsic Liouville field. Their string theory has the space supersymmetry which corresponds roughly to the supersymmetry in the transverse direction. It would be interesting to redo their calculation à la Polchinski and Strominger and see whether it is equivalent to the noncritical GS string. The main difference from our construction of the RNS string is their insistence on global $N=2$ world sheet supersymmetry.

D. As pointed out by Hughes and Polchinski, there can be a supersymmetric vortex with the right-left imbalance of the world sheet fermion content. See also [19. Such a string is heterotic 20]. The Lorentz anomaly on the world sheet should be cancelled by some additional fermions, as in the critical case. The conformal gauge approach to non-critical heterotic string has been worked out in [21]. There should be no problem in applying it to an effective heterotic string.

E. The fact that the two formulations of superstrings are different in general raises a similar question concerning super p-branes [22]. In fact, a supersymmetric generalization à la RNS must include the Einstein term 23]. It is not clear whether it is equivalent to the GSlike supermembranes. Semiclassical quantization in light-cone gauge of supermembranes is discussed in [24].

F. Polchinski and Strominger have made a nice analog of the effective string theory to low energy pion dynamics. Here we make another analog, this time with $4 D$ Einstein gravity. The general relativity is based on purely geometrical concept, is non-renormalizable, and makes sense only when we expand around a non-singular background. These are the features of effective string theories too. In both cases one can add higher derivative terms to make the theories renormalizable, but at the price of giving up (perturbative) unitarity (for classical statistical mechanics of a membrane, this is not a problem). Moreover, both theories allow supersymmetric generalization. It is perhaps then adequate to take the ef- 
fective string theory as a laboratory for quantum gravity. For example, it is possible that the continuum limit of an effective string theory involves an understanding of expansion around the unbroken vacuum $\partial_{\alpha} X_{c l}=0$. This in turn might teach us something about the unbroken phase 25] of general relativity and fundamental string theory.

In conclusion, we have quantized the effective superstrings. The RNS and GS strings are different in non-critical dimensions. We have also pointed out some formal analogies with 4D (super)gravity.

\section{Acknowledgement}

I would like to thank Joe Polchinski for discussions. This work is supported in part by U.S. Department of Energy Contract No. DE-AC02-76ER13065.

\section{References}

[1] J. Polchinski and A. Strominger, Texas preprint UTTG-17-91.

[2] P. Di Vecchia and S. Ferrara, Nucl. Phys. B130 (1977) 93.

[3] J. Hughes and J. Polchinski, Nucl. Phys. B278 (1986) 147.

[4] M.B. Green and J.H. Schwarz, Phys. Lett. 136B (1984) 367.

[5] See, e.g., V.S. Dotsenko, Nucl. Phys. B285 (1987) 45.

[6] S. Deser and B. Zumino, Phys. Lett. 65B (1976) 369;

L. Brink, P. Di Vecchia and P. Howe, Phys. Lett. 65B (1976) 471.

[7] A.M. Polyakov, Phys. Lett. 103B (1981) 211.

[8] E. Martinec, Phys. Rev. D28 (1983) 2604. 
[9] F. David, Mod. Phys. Lett. A3 (1988) 1651;

J. Distler and H. Kawai, Nucl. Phys. B321 (1989) 509.

[10] J. Distler, Z. Hlousek and H. Kawai, Int. J. Mod. Phys. A5 (1990) 391.

[11] P. van Nieuwenhuizen, Phys. Rep. 68 (1981) 189.

[12] F. Gliozzi, J. Scherk and D. Olive, Nucl. Phys. B122 (1977) 253.

[13] See, e.g., M.B. Green, J.H. Schwarz and E. Witten, Superstring Theory, Cambridge (1987).

[14] S. Carlip, Nucl. Phys. B195 (1987) 365.

[15] R.E. Kallosh and A.Y. Morozov, Int. J. Mod. Phys. A3 (1988) 1943.

[16] W. Siegel, Phys. Lett. 128B (1983) 397.

[17] E. Witten, in Fourth Workshop on Grand Unification, ed. P. Langacker et al., Birkhauser (1983).

[18] D. Kutasov and N. Seiberg, Phys. Lett. 251B (1990) 67.

[19] J.A. Harvey, Cargese Lecture (1987).

[20] D.J. Gross, J.A. Harvey, E. Martinec and R. Rohm, Phys. Rev. Lett. 54502.

[21] Z. Yang, unpublished (1989).

[22] J. Hughes, J. Liu and J. Polchinski, Phys. Lett. 180B (1986) 370.

[23] P.S. Howe and R.W. Tucker, J. Phys. A10 (1977) L155. 
[24] M.J. Duff, T. Inami, C.N. Pope, E. Sezgin and K.S. Stelle, Nucl. Phys. B297 (1988) 515.

[25] E. Witten, Phil. Trans. R. Soc. Lond. A329 (1989) 349. 